\title{
MBD5 haploinsufficiency is associated with sleep disturbance and disrupts circadian pathways common to Smith-Magenis and fragile $X$ syndromes
}

\author{
Sureni V Mullegama ${ }^{1}$, Loren Pugliesi ${ }^{2}$, Brooke Burns ${ }^{2}$, Zalak Shah ${ }^{2}$, Raiha Tahir ${ }^{2}$, Yanghong Gu${ }^{1}$, \\ David L Nelson ${ }^{1}$ and Sarah H Elsea ${ }^{\star, 1,2}$
}

Individuals with autism spectrum disorders (ASD) who have an identifiable single-gene neurodevelopmental disorder (NDD), such as fragile X syndrome (FXS, FMR1), Smith-Magenis syndrome (SMS, RAl1), or 2q23.1 deletion syndrome (del 2q23.1, MBD5) share phenotypic features, including a high prevalence of sleep disturbance. We describe the circadian deficits in del 2q23.1 through caregiver surveys in which we identify several frequent sleep anomalies, including night/early awakenings, coughing/ snoring loudly, and difficulty falling asleep. We couple these findings with studies on the molecular analysis of the circadian deficits associated with haploinsufficiency of MBD5 in which circadian gene mRNA levels of NR1D2, PER1, PER2, and PER3 were altered in del 2q23.1 lymphoblastoid cell lines (LCLs), signifying that haploinsufficiency of MBD5 can result in dysregulation of circadian rhythm gene expression. These findings were further supported by expression microarrays of MBD5 siRNA knockdown cells that showed significantly altered expression of additional circadian rhythm signaling pathway genes. Based on the common sleep phenotypes observed in del 2q23.1, SMS, and FXS patients, we explored the possibility that MBD5, RAI1, and FMR1 function in overlapping circadian rhythm pathways. Bioinformatic analysis identified conserved putative E boxes in MBD5 and RAI1, and expression levels of NR1D2 and CRY2 were significantly reduced in patient LCLs. Circadian and mTOR signaling pathways, both associated with sleep disturbance, were altered in both MBD5 and RAl1 knockdown microarray data, overlapping with findings associated with FMR1. These data support phenotypic and molecular overlaps across these syndromes that may be exploited to provide therapeutic intervention for multiple disorders.

European Journal of Human Genetics (2015) 23, 781-789; doi:10.1038/ejhg.2014.200; published online 1 October 2014

\section{INTRODUCTION}

Autism spectrum disorder (ASD; MIM 209850) is a neurodevelopmental disorder (NDD) that affects all areas of a child's development, including social interaction, communication, and behavior. Approximately $10 \%$ of children with ASD have an identifiable genetic condition and may present with deficits in language, cognition, motor function, speech impairment, intellectual disability (ID), or seizures. ${ }^{1}$ In addition, behavioral phenotypes such as repetitive, stereotypical, or problematic behaviors are often present.

Significant sleep problems are highly prevalent in individuals with ASD. ${ }^{2}$ Parents of children with ASD report $50-80 \%$ prevalence of sleep problems compared with a $9-50 \%$ prevalence rate reported by parents of age-matched typically developing children. ${ }^{2}$ Common sleep etiological factors that are present in children with ASD are rapid eye movement sleep abnormalities, dysregulation of melatonin synthesis, difficulty in settling to sleep, night waking, irregular sleep patterns, short-duration sleep, and daytime sleepiness. ${ }^{2,3}$ Persistent sleep problems are thought to adversely affect cognitive, physical, and social function, learning, mood, and behavior in these individuals and, in addition, cause significant stress for families and caretakers. ${ }^{3}$ Better understanding of the sleep difficulties experienced by children with ASD may reduce the adverse effects and decrease family stress, possibly with targeted therapies.
About $10-15 \%$ of single-gene NDDs, such as fragile X syndrome (FXS; MIM 300624; FMR1, MIM 309550), Rett syndrome (RTT; MIM 312750; MECP2, MIM 300005), and Smith-Magenis syndrome (SMS, MIM 182290; RAI1, MIM 607642), are associated with a high prevalence of autism. ${ }^{4}$ Further, these NDDs share many overlapping features, including a high prevalence of sleep disturbance. Understanding the molecular alterations in NDDs individually and collectively and the subsequent overlapping pathways affected that lead to the sleep disturbance could possibly lead to behavioral and pharmaceutical therapies.

The 2q23.1 deletion syndrome (del 2q23.1) (MIM 156200) is a NDD/ASD that results from haploinsufficiency of a common gene in the del 2q23.1 region called methyl-CpG-binding domain 5 (MBD5) (MIM 611472). ${ }^{4}$ Genomic disruptions of MBD5 lead to the primary clinical phenotypes present in the disorder, including ID, epilepsy, speech impairment, unusual behaviors, ASD, and broad-based ataxic gate. ${ }^{4}$ Sleep disturbance have been previously reported in a few cases. ${ }^{4-12}$ Some of the sleep disturbances mentioned in these cases are waking 6-8 times per night, apparent night terrors in the early part of sleep, and waking in the early hours of the morning. ${ }^{4-12}$ Although it is clear a sleep disturbance exists in del 2q23.1, no systematic characterization of the extent of sleep problems in this population has been published.

\footnotetext{
${ }^{1}$ Department of Molecular and Human Genetics, Baylor College of Medicine, Houston, TX, USA; ${ }^{2}$ Department of Human and Molecular Genetics, Virginia Commonwealth University School of Medicine, Richmond, VA, USA

${ }^{*}$ Correspondence: Dr SH Elsea, Department of Molecular and Human Genetics, Baylor College of Medicine, One Baylor Plaza, NAB2015, Houston, TX 77030, USA. Tel: +1 713798 5484; Fax: +1 832825 1269; E-mail: elsea@bcm.edu
}

Received 12 March 2014; revised 23 July 2014; accepted 26 August 2014; published online 1 October 2014 
We report clinical characterization of the circadian deficits in the del 2q23.1 syndrome and couple this analysis with identification of the molecular deficits associated with haploinsufficiency of MBD5. We investigated circadian gene expression in del 2q23.1 in comparison to that present in SMS and FXS, illustrating that circadian gene dysregulation is a significant etiology in these disorders. Furthermore, we examined whether circadian rhythm pathways and mammalian target of rapamycin (mTOR) pathways, which are thought to be responsible for circadian deficits, are altered in these disorders. ${ }^{13}$

\section{MATERIALS AND METHODS}

\section{Participants for sleep survey}

Potential participants were contacted via the 2q23.1 Facebook support group page and the online support group, www.2q23.org, where online surveys were posted. Emails with a brief description of the survey and the link to the online survey were sent to clinical geneticists and genetic counselors to spread awareness of the study. Surveys were advertised and open for collection from mid-November through December 2012.

Participants of the sleep study consisted of 19 children between 9 months and 11 years with a molecular diagnosis of del 2q23.1. Parents served as participants in completing the surveys. School-age children as described by the National Sleep Foundation (5-12 years) comprised $58 \%$ of the study population (mean $=5.5$ years). The majority of the children were from middle socioeconomic backgrounds and were predominantly white. Males (43.8\%) and females $(56.2 \%)$ were represented. The mean age of diagnosis was 3.9 years $(\mathrm{SD}=2.136$ years $)$. Caregivers/parents reported that their child's diagnosis of 2q23.1 deletion syndrome was confirmed using chromosomal microarray ( 53\%), FISH $(\sim 21 \%)$, karyotype $(\sim 16 \%)$, and MBD5 mutation analysis ( $6 \%)$. The majority of the survey participants were mothers $(84.2 \%)$, had a college education or greater (68.4\%), and either worked part-time or did not work outside the home (63.1\%). Families did not receive any incentive for taking part in the study. All information was collected after informed consent was obtained and in accordance with local institutional review board protocols from Virginia Commonwealth University (VCU).

\section{Demographic and sleep questionnaires}

Demographic and sleep questionnaires were created and administered using SurveyMonkey (https://www.surveymonkey.com/; Palo Alto, CA, USA). Demographic information consisted of nine questions, including participant's relationship to child, gender, highest level of education, employment status, and occupation, and child's age at diagnosis, diagnosis, current age, and gender. The sleep questionnaire was designed to assess questions regarding child sleep patterns, behaviors, and sleep troubles experienced and was comprised of select questions from the Pittsburgh Sleep Quality Index (PSQI), the Epworth Sleepiness Scale (ESS), and additional questions regarding specific sleep concerns and behaviors. The PSQI is a self-rated questionnaire designed to assess the sleep disturbance and overall sleep quality over a 1-month time interval. ${ }^{14}$ The ESS is a standard measure that assesses the level of daytime sleepiness and thus the likelihood of dozing off during common sedentary daytime activities. ${ }^{15}$ Based on response, a total score can range from 0 to 24. A score of $\geq 10$ represents 'excessive daytime sleepiness'. ${ }^{15}$ The additional questions surveyed specific sleep and nighttime behaviors of the child, including wandering, food seeking, sleepwalking, bedtime, and waking difficulties, naps, and sleepiness at school.

\section{Cell culture}

Lymphoblastoid cell lines (LCLs) were created from blood samples collected after informed consent was obtained and in accordance with the local institutional review board-approved protocols from VCU or Baylor College of Medicine (BCM). At least three LCLs from del 2q23.1, SMS, FXS, and controls were cultured as previously described. ${ }^{8}$ Furthermore, previously published LCL controls were used. ${ }^{8}$ LCLs utilized in this study included the following: SMS367 ${ }^{8}$ (SCV000172263) with a single-copy loss of $204 \mathrm{~kb}$ at chr(2) (q23.1), chr2.hg18:g.(148,447,295_148,651,456)del; SMS185 ${ }^{8}$ (SCV000172261) with a single-copy loss of $930 \mathrm{~kb}$ at $\operatorname{chr}(2)$ (q23.1), chr2.hg18:g.(148,447,496 _149,377,297)del; SMS361 ${ }^{4}$ (SCV000172262) with a single-copy loss of $3.51 \mathrm{Mb}$ at $\operatorname{chr}(2)$ (q22.3-q23.3), chr2.hg18:g.(146,798,229_150,310,317)del; SMS129 ${ }^{16,17}$ (RCV000003082.1) with a 29-basepair deletion in the coding region of RAI1, NM_030665.3:c.2773_2801del; SMS175 ${ }^{18}$ (rs104894634, RCV000003084.1) with an RAI1 mutation, NM_030665.3:c.4685A>G; SMS123 (SCV000172264), ${ }^{19}$ with a typical SMS 17p11.2 deletion, chr17:g.(AC027266_AL353996)_ (AC015726_AC087393)del; SMS105 (SCV000172265), ${ }^{19}$ with a typical SMS 17p11.2 deletion, chr17:g.(AC027266_AL353996)_(AC015726_AC087393)del; FXS1 (SCV000172267), ${ }^{20}$ with a fully expanded CGG repeat in FMR1 (GM09237, Coriell Cell Repositories, Camden, NJ, USA), (NM_002024.5)c.158GGC(950); FXS2 (SCV000172266) ${ }^{21}$ with an expanded CGG repeat in FMR1 (NM_002024.5)C.-158GGC(500); and FXS3 (SCV000172267) ${ }^{22}$ with a deletion of FMR1 exons 1-7, chrX.hg18:g.(146,703,942_146,820,448)del. All variant information is available in either dbSNP (http://www.ncbi.nlm.nih.gov/ SNP/) or ClinVar (http://www.ncbi.nlm.nih.gov/clinvar/). Neuroblastoma cell line, SH-SY5Y, was cultured according to the standard protocols (ATCC CRL-2266, Manassas, VA, USA). Human embryonic kidney cell line, HEK293T, was cultured according to the standard protocols (ATCC CRL-11268).

\section{Quantitative real-time PCR}

Total RNA was isolated from SH-SY5Y cells and 2q23.1 deletion syndrome, SMS, FXS, and control LCLs, via Trizol (Invitrogen, Carlsbad, CA, USA) according to the standard protocols. RNA was quantified using the NanoDrop ND-100 Spectrophotometer (NanoDrop Technologies, Inc., Wilmington, DE, USA). First-strand cDNA synthesis was performed using the qScript cDNA SuperMix (Quanta BioScience, Gaithersburg, MD, USA) according to the manufacturer's instructions. For quantitative real-time PCR, predesigned Taqman MGB probes from Assays-on-Demand Gene Expression Products (ABI, Applied Biosystems, Life Technologies Inc., Carlsbad, CA, USA) were used for all genes in the study (Supplementary Table S1). All samples were run and analyzed according to previously published methods using either ABI Prism 7900 HT Sequence Detection System (Life Technologies Inc.) or BioRad CFX Connect Real-Time PCR Detection System (Bio-Rad Laboratories, Inc., Hercules, CA, USA). ${ }^{23}$ GAPDH (MIM 138400, Hs9999905_ml) mRNA served as an endogenous control.

\section{siRNA knockdown of MBD5 and RAI1 in HEK293T and SH-SY5Y cells}

Methodology for knockdowns of MBD5 and RAI1 were performed in HEK293T cells as previously described. ${ }^{24}$ Knockdown of MBD5 or RAI1 was performed according to the standard protocol in SH-SY5Y using Silencer Select predesigned short interfering RNAs, which give the highest knockdown and lowest off-target effects, specific to MBD5 (siRNA ID no. s31485) or RAI1 (siRNA ID no. s21107) were designed to target constitutive exons found in human MBD5 and RAI1 transcripts. A scrambled siRNA (siRNA ID no. 4390849) served as the negative control and GAPDH siRNA (siRNA ID no 4390849) was used in control experiments to test the optimization of the siRNA transfection protocol and were performing according to the manufacturer's instructions (Ambion, Austin, TX, USA) (Cat. no. 4392420 and Cat. no. 4392420). Each transfection was performed in quadruplicate, and three biological replicates were used for array studies and gene expression analysis, while the remaining sample was used for further expression studies.

\section{Microarray}

Three biological replicates from the following treatment groups from SH-SY5Y cells (MBD5 knockdown (KD), RAI1 KD, scrambled siRNA) were sent for microarray analysis. Microarray hybridizations were performed at the VCU Massey Cancer Center Nucleic Acids Research Facility, DNA Microarray Core, using an Illumina HumanWG-12 v4.0 expression beadchip (Illumina, San Diego, CA, USA) (containing $>47000$ probes) and were read and analyzed according to previously published methods. ${ }^{25}$ Briefly, array was read using an Illumina BeadScan confocal scanner and analyzed by the Illumina's BeadStudio software. The data were normalized by quantile method using the Illumina BeadStudio Software. $P$-values $<0.05$ were used to determine which probes were detected. One-way ANOVA was subsequently performed comparing control versus MBD5 KD or RAI1 KD groups to identify differentially expressed genes. The criterion for a gene to be differentially expressed was set at $P \leq 0.05$ and then at $P \leq 0.01$. 
Ingenuity Pathway Analysis (IPA)

IPA was used to identify gene networks and pathways and likely upstream regulators that were significantly altered in knockdown cells than in controls. Significant interactions were determined using the Ingenuity Pathway Knowledge Base and a Fisher's exact test to calculate a $P$-value determining the probability that each function network or pathway assigned to that data set is due to chance alone. Molecules from the data set that met the $P<0.05$ cutoff and that were associated with biological functions in the Ingenuity knowledge Base were considered for the analysis.

\section{E box location in MBD5, RAI1 and FMR1 and E box conservation analysis}

A Perl program was written to look for the nucleotide sequences that represent the E box, 'CANNTG', where 'NN' can be any nucleotide. This E box sequence was searched for starting 2500 bases upstream of the ATG for FMR1, RAI1, or MBD5. Each E box sequence identified in each gene was then aligned to the mouse counterpart to determine evolutionary conservation using BLAST from NCBI (http://blast.ncbi.nlm.nih.gov/Blast.cgi). In order to validate this approach, we also investigated several non-circadian genes, including $B M P 7$, GATA4, TBX5, HAND2, and NOG, which are involved in bone growth and development, and looked for E box sequences upstream 2500 bases from the ATG. We found that these genes have either 0 or $1 \mathrm{E}$ boxes compared with MBD5 and RAI1, which have a larger count of $\mathrm{E}$ boxes as discussed in the Results. We also looked at known biologically confirmed circadian rhythmdependent genes such as SNAI1, SMAD3, WEE1, OTX2, and TSHB and found they also have a greater count of E boxes ( $>3$ ), similar to RAI1 and MBD5.

\section{Statistical analyses}

Statistical analysis for gene expression data was performed with Prism 4 version 4.0b (GraphPad Software, San Diego, CA, USA), as previously described. ${ }^{4,25}$

\section{RESULTS}

Frequency and nature of sleep problems in children with 2 q23.1 deletion syndrome

Surveys of parents and caregivers of children with del 2q23.1 demonstrated a variety of sleep anomalies affecting these individuals (Table 1). Among the school-age children (age 5-12 years), 45\% reported having 'fairly bad' sleep. Among these school-age children, parents considered the sleep problems present to be associated with behaviors they observed (Table 2). Supporting these concerns, parents reported that $33 \%$ of children aged 5-12 years were napping, a rate higher than expected for school-age children. As parents reported that many of their children were not sleeping well throughout the night, an ESS score was calculated to assess each child's daytime sleepiness (see Methods). Total scores ranged from 4 to 17 (mean $=9.4$, SD 3.99), which was 0.6 points below the threshold of 10 for 'excessive daytime sleepiness', as defined by the ESS among school-age children. Of note, $43.7 \%$ of the children surveyed held a score of $\geq 10$, indicating that a substantial portion of the children with del 2 q23.1 have excessive daytime sleepiness likely due to poor and inconsistent sleep at night. Finally, many parents reported using various methods to ensure proper sleep for their children. The most commonly used interventions were regular naps for their children (62.50\%) and use of containment (enclosed beds, locked doors) to prevent their child from wandering at night $(66.7 \%)$. However, many parents reported using daily pharmacological (melatonin, clonidine, and trazadone) interventions to cope with the sleep disturbances (55.6\%), indicating a significant impact on the family.

\section{Dysregulation of circadian genes in del 2q23.1 syndrome LCLs}

Given that individuals with del 2q23.1 have a sleep phenotype, we sought to determine whether circadian gene expression was affected, perhaps pointing to an etiology for the sleep disturbance. We tested the expression of MBD5 to confirm MBD5 haploinsufficiency in the del 2q23.1 cell lines, which was consistent with previous studies (Figure 1). ${ }^{26}$ Based on literature indicating altered expression of circadian genes in SMS LCLs, we tested the expression of four circadian genes, NR1D2, PER1, PER2, and PER3, in 2q23.1 deletion syndrome LCLs that were grown at the same time and under the same conditions. ${ }^{23}$ The transcript levels of the above four circadian rhythm genes had significantly reduced mRNA expression in del 2q23.1 LCLs (Figure 1). Other methyl CpG-binding domain (MBD) genes (MBD2, $M B D 3$, and $M B D 4$ ) were tested as non-affected gene controls, and no differences were found in the mRNA levels of these genes (Figure 1).

\section{Circadian rhythm gene expression altered by haploinsufficiency of MBD5}

Given the association of sleep disturbance and altered circadian gene expression in del 2q23.1 patients, we hypothesized that MBD5 can regulate additional circadian genes and that haploinsufficiency of MBD5 causes dysregulation of the circadian rhythm pathway. Thus, through microarray technology, we investigated the effect of knocking down MBD5 for 24 and $32 \mathrm{~h}$ with siRNA to levels that mimic expression seen in del 2q23.1 patients ( $\sim 50-60 \%$ of normal expression) on circadian gene expression in SH-SY5Y cell lines. ${ }^{4,26}$ We first knocked down MBD5 in HEK293T cells to test the efficacy in knocking down MBD5 and then proceeded to SH-SY5Y lines, which are less robust and harder to transfect. Real-time qRT-PCR showed $\sim 60 \%$ knockdown of MBD5 was achieved in HEK293T and SH-SY5Y cells (Supplementary Figure S1). In the microarray data on KD of

Table 1 Parent-reported sleep concerns in 2q23.1 deletion syndrome

\begin{tabular}{|c|c|c|c|c|}
\hline \multirow[b]{2}{*}{ Sleep concern } & \multicolumn{2}{|c|}{$\%$} & \multirow[b]{2}{*}{$\begin{array}{c}\text { Less than } \\
\text { once a month }\end{array}$} & \multirow[b]{2}{*}{$\begin{array}{c}\text { Not during the } \\
\text { past month }\end{array}$} \\
\hline & $\begin{array}{l}\text { Three or more } \\
\text { times a week }\end{array}$ & $\begin{array}{c}\text { Once or twice per } \\
\text { past month }\end{array}$ & & \\
\hline Cannot get to sleep within $30 \mathrm{~min}$ & 25 & 42.8 & 6.3 & 25 \\
\hline Wake up in the middle of the night or early morning & 73.3 & 20 & 0 & 6.7 \\
\hline Cannot breathe comfortably & 12.5 & 25 & 6.3 & 56.3 \\
\hline Cough or snore loudly & 43.8 & 12.5 & 0 & 43.8 \\
\hline Feel too cold & 12.5 & 18.8 & 6.3 & 62.5 \\
\hline Feel too hot & 18.8 & 6.3 & 0 & 75 \\
\hline Had bad dreams & 6.3 & 18.8 & 18.8 & 56.3 \\
\hline Other ${ }^{\mathrm{a}}$ & 46.2 & 0 & 0 & 53.8 \\
\hline
\end{tabular}

ancludes bed wetting, toileting, agitation, and nightmares. 
Table 2 Sleep behaviors of school-age children with 2 q23.1 deletion syndrome

\section{Behavior}

Wanders at night

Seeks food

Sleep walks

Difficulty putting to bed

Child naps on their own
Difficult to wake

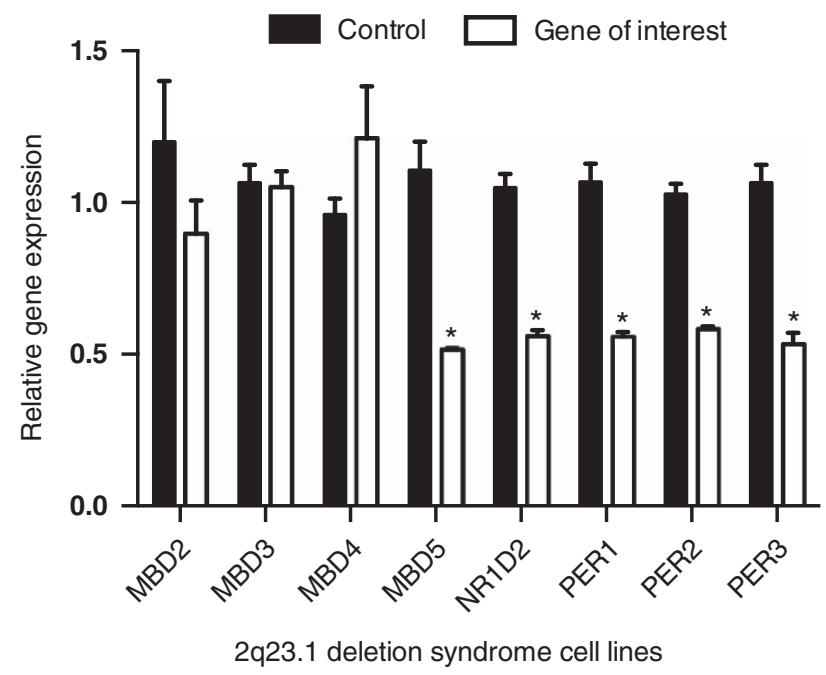

Figure 1 Altered expression of circadian genes in 2q23.1 deletion syndrome. Quantitative real-time PCR was performed on LCLs from normal controls and from individuals with 2q23.1 deletion syndrome, as described in the Methods. ${ }^{4}$ Expression levels of key circadian genes (NR1D2, PER1, PER2, $P E R 3)$ were evaluated. Data are an average of three independent experiments performed in triplicate. Haploinsufficiency of MBD5 was confirmed in these patient samples. Expression levels of $M B D 2, M B D 3$, and $M B D 4$ served as non-affected gene controls, illustrating normal levels of expression in these LCLs. Quantification of the test gene was compared with that of the housekeeping gene GAPDH. All expression values were calculated relative to control levels set at 1.0. Error bars represent the SEM. A Pvalue $<0.005$ was determined by one-sample $t$-tests. Asterisks indicate significant differences in gene expression.

MBD5 in SH-SY5Y, 1903 genes were significantly upregulated $(P<0.05)$; using a higher stringency $(P<0.01), 619$ genes were significantly upregulated. Furthermore, in $M B D 5$ knockdown array data, 1880 genes were significantly downregulated $(P<0.05)$; using a high stringency $(P<0.01) 1110$ genes were significantly downregulated. The microarray revealed significant changes of mRNA abundance for 63 genes implicated in control of circadian rhythm $(P<0.05)$, including CLOCK, PER3, and RAI1 (Table 3). The top 16 significantly dysregulated circadian rhythm genes can be seen in Table $3(P \leq 0.001)$. Using real-time qRT-PCR, we confirmed that mRNA expression levels of several of these genes were significantly altered in SH-SY5Y knockdown cells, validating the microarray data (Figure 2a). Importantly, the expression of CLOCK, a key component of the mammalian circadian oscillator that transcriptionally regulates many key circadian genes was significantly reduced to $\leq 50 \%$ of control (Figure 2a). Other essential components of the circadian feedback loop PER1, PER3, CRY1, CRY2, NR1D1 and NR1D2 were significantly downregulated in MBD5 knockdown cells (Figure 2a). Other CLOCK-related genes, ATF2 and ATF4, were significantly overexpressed compared with the control (Figure 2a). IPA confirmed the observation that the circadian rhythm pathway was significantly dysregulated due to MBD5 knockdown ( $P=0.00474$; Figure $2 \mathrm{~b}$ ).

\section{Effect of MBD5, RAI1, and FMR1 on circadian rhythm gene expression}

Based on the sleep phenotype observed in del 2q23.1, SMS, and FXS, we hypothesized that MBD5, RAI1, and FMR1 function in common or overlapping pathways involved in circadian rhythm, which when disrupted lead to the common sleep disturbances present in the above disorders. We analyzed the mRNA expression of key circadian genes MBD5, RAI1, and FMR1 in the three syndromes in three patientderived LCLs for each syndrome compared with three normal controls (Figure 3a-h). FXS cell lines showed normal expression for all PER genes; however, the mRNA levels of PER1, PER2, and PER3 were significantly reduced $(<60 \%)$ in the del 2 q23.1 and SMS cell lines (Figure $3 \mathrm{a}-\mathrm{c}$ ). mRNA levels of NR1D2 and CRY2 were also significantly reduced $(<60 \%)$ in cell lines from all three syndromes (Figure $3 \mathrm{~d}-\mathrm{e}$ ). CLOCK and CRY1 were evaluated, but the expression was too low to effectively assay in LCLs. Overall, these data alert us to a possible molecular basis for the similar phenotypes observed across these three disorders.

Due to similar mRNA expression patterns of circadian rhythm genes in LCLs from del 2q23.1, SMS, and FXS, we wanted to assess the relationships between mRNA expression of MBD5, RAI1, and FMR1 in del 2q23.1, SMS, and FXS LCLs. As expected, each disease gene had reduced expression in its respective syndrome (Figure $3 \mathrm{f}-\mathrm{h}$ ). Interestingly, MBD5 expression was significantly increased in FXS cell lines $(P<0.01)$, suggesting that $F M R 1$ is critical for proper dosage of MBD5 (Figure 3f). RAI1 expression was significantly reduced in cells from del 2q23.1 subjects $(P<0.0001)$, which suggests that RAI1 may function downstream of MBD5 and could be either directly or indirectly regulated by MBD5 (Figure 3f). Further, expression of FMR1 was increased in del 2q23.1 LCLs $(P<0.02)$ (Figure 3f). Based on these findings, we propose that MBD5, RAI1, and FMR1 function in an overlapping circadian network, with NR1D2 and CRY2 having important roles in linking these disorders together and contributing to overlapping sleep phenotypes. Overall, data demonstrate the possibility that these genes are involved in common circadian pathways.

\section{Identification of potential $\mathrm{E}$ boxes in MBD5 and RAI1}

E boxes, which are found in the promoter or enhancer regions, are thought to have an important role in the regulation of the mammalian circadian clock and clock genes. ${ }^{27}$ Nine closely connected circadian rhythm genes have E boxes, including CLOCK, NPAS2, ANTL, CRY1, BHLHB2, PER1, PER2, and PER3. As MBD5, RAI1, and FMR1 participate in circadian regulation, we sought to determine whether E boxes were also present in the promoter or enhancer regions, which would suggest that these genes are circadianly regulated through their E boxes. Assessment of $\sim 2500$ nucleotides upstream of each ATG translation start site revealed the presence of putative $\mathrm{E}$ boxes in each gene (Table 4). We then investigated whether these $\mathrm{E}$ boxes were evolutionarily conserved in non-coding regions of Rail, Mbd5, and Fmr1 in mice (Supplementary Figure S2), as these genes are conserved between human and mice. None of the E boxes of human FMR1 were identified in Fmrl promoter or enhancer region. Only one E box in human RAI1 was found in mouse Rail, while all of the E boxes found in MBD5 were found in Mbd5 (Supplementary Figure S2). 
Table 3 Circadian rhythm genes dysregulated due to MBD5 haploinsufficiency in SH-SY5Y cells

\begin{tabular}{|c|c|c|c|}
\hline Gene symbol & Fold change & Name & Cellular function \\
\hline \multicolumn{4}{|l|}{ Upregulated } \\
\hline ATF2 & 2.0396 & Activating transcription factor 2 & Transcription factor, member of the leucine zipper family of DNA-binding proteins \\
\hline MAPK1 & 1.540 & Mitogen-activated protein kinase 1 & $\begin{array}{l}\text { Integration point for multiple biochemical signals that are involved in a wide variety of cellular } \\
\text { processes, such as proliferation, differentiation, transcription regulation, and development }\end{array}$ \\
\hline CSNK1E & 1.371 & Casein kinase 1 , epsilon & Role in regulating the phosphorylation and abundance of per proteins in animals \\
\hline ATF4 & 1.2486 & Activating transcription factor 4 & Transcription factor, member of the leucine zipper family of DNA-binding proteins \\
\hline PRNP & 1.121 & Prion protein & Membrane glycosylphosphatidylinositol-anchored glycoprotein \\
\hline \multicolumn{4}{|l|}{ Downregulated } \\
\hline$R O R B$ & -0.0357 & RAR-related orphan receptor beta & Transcription factor possibly related to the circadian pacemaking system \\
\hline CRY1 & -1.0362 & Cryptochrome 1 & Key component of the circadian core oscillator complex \\
\hline CLOCK & -1.1599 & Circadian locomotor output cycles kaput & $\begin{array}{l}\text { Encodes a basic helix-loop-helix (bHLH)-PAS transcription factor that is essential for circadian } \\
\text { rhythm }\end{array}$ \\
\hline$R A / 1$ & -1.227 & Retinoic acid induced 1 & Positive transcriptional regulator of $C L O C K$ and possible role in circadian oscillator \\
\hline CRY2 & -1.3004 & Cryptochrome 2 & Central to the core autoregulatory loop of the mammalian circadian clock \\
\hline$N R 1 D 1$ & -1.3401 & $\begin{array}{l}\text { Nuclear receptor subfamily } 1 \text {, group } D \text {, mem- } \\
\text { ber } 1 / \text { nuclear receptor Rev-ErbA-alpha }\end{array}$ & Major regulator of cyclic BMAL1 transcription \\
\hline JUNB & -1.377 & Jun B proto-oncogene & Role in regulation of light-induced gene expression in the suprachiasmatic nucleus \\
\hline PER3 & -1.4271 & Period circadian protein homolog 3 & Maintenance of circadian rhythm in cells \\
\hline NOS2 & -1.490 & Nitric oxide synthase 2 , inducible & $\begin{array}{l}\text { Biologic mediator in several processes, including neurotransmission and antimicrobial and } \\
\text { antitumor activities }\end{array}$ \\
\hline NR1D2 & -1.544 & $\begin{array}{l}\text { Nuclear receptor subfamily } 1 \text {, group } D \text {, } \\
\text { member } 2 / \text { nuclear receptor Rev-ErbA-beta }\end{array}$ & $\begin{array}{l}\text { Functions as a transcriptional repressor and may have a role in circadian rhythms and } \\
\text { carbohydrate and lipid metabolism }\end{array}$ \\
\hline PER1 & -2.3391 & Period circadian protein homolog 1 & Maintenance of circadian rhythm in cells \\
\hline
\end{tabular}

\section{MBD5 and RAI1 are involved in molecular pathways related to sleep}

Based on the more common sleep phenotype and our previous mRNA expression studies, we have shown that there is a relationship between MBD5 and RAI1. We further investigated the hypothesis that MBD5 and RAI1 have shared molecular pathways that when altered cause some aspects of the sleep phenotype present in del 2q23.1 and SMS patients.

To test this hypothesis, we used our microarray knockdown data for MBD5 and RAI1 with IPA. We knocked down MBD5 or RAI1 using siRNA technology in SH-SY5Y cell lines (Supplementary Figure S3) and determined genome-wide levels of mRNAs using microarrays. In the microarray data on KD of RAI1 in SH-SY5Y, 1358 genes were significantly upregulated $(P<0.05)$; using a higher stringency $(P<0.01), 836$ genes were significantly upregulated. Furthermore, in the RAI1 knockdown array data, 1459 genes were significantly downregulated $(P<0.05)$; using a high stringency $(P<0.01) 349$ genes were significantly downregulated. IPA demonstrated that mRNAs for genes in two pathways related to sleep were altered in both knockdown studies. The first pathway is circadian rhythm signaling $(P=0.00329)$, where 63 mRNAs showed significantly altered abundance in both MBD5 and RAI1 knockdowns $(P<0.05)$. The mTOR signaling pathway also showed significant changes in both knockdowns $(P=0.0304)$ (Figure 4a). Four mRNAs in the mTOR pathway were increased (PLD (MIM 602382), ULK1 (MIM 603168), eIF4G (MIM 600495), eIF3 (MIM 602039)), and three showed reduced levels (REDD1 (MIM 607729), REHB (MIM 601293), and VEGF (MIM 192240)). Analysis of the mRNA expression level of $m T O R$ in del 2q23.1 and SMS LCLs showed a significant increase in expression compared with normal controls (Figure $4 \mathrm{~b}$ ), which corroborated our microarray studies. These data support a functional role for MBD5 and RAI1 in the same pathways contributing to the sleep phenotype present in the associated disorders.

\section{DISCUSSION}

Proper circadian rhythms are vital to human physiological processes, including body temperature, feeding behavior, drug and xenobotic metabolism, glucose homeostasis, cell cycle progression, learning, memory, and development. ${ }^{28}$ The mammalian circadian clock is composed of a core set of genes that are involved in cell autonomous transcription-translation feedback loops, including CLOCK, NPAS2, BMAl1, PER genes (1-3), CRY genes (1-2), NR1D genes (1-2), ROR genes $(A-C)$, and a number of candidate clock components whose roles remain to be more clearly defined. The disruption of these genes is now thought to influence a range of disease-related pathways. ${ }^{28}$ Defects in circadian rhythm may also affect the daily timing of neurobehavioral and endocrine functions. ${ }^{29}$ Circadian rhythm defects seen in ASD are thought to contribute not only to the sleep disturbances but also to behavior, learning, and other phenotypes seen in these patients. ${ }^{29}$

\section{2q23.1 deletion syndrome, $M B D 5$, and sleep}

In this study, we show that individuals with del 2q23.1 syndrome have sleep disturbances. These sleep studies further support previous reports regarding del 2q23.1 patients' sleep habits. ${ }^{4-12}$ Although this survey may reflect a subjective measure of the sleep phenotype present in 2q23.1 deletion syndrome patients as parents/guardians answered the survey, parents are in a unique position to describe their child's regular sleep behaviors. Importantly, $\sim 55.6 \%$ of parents reported that they use medication to improve the poor sleep patterns in their children, which is a significant indicator of the difficulties faced by the child and the family because of the sleep problems. As the majority of children were taking sleep medication during the survey, this may 


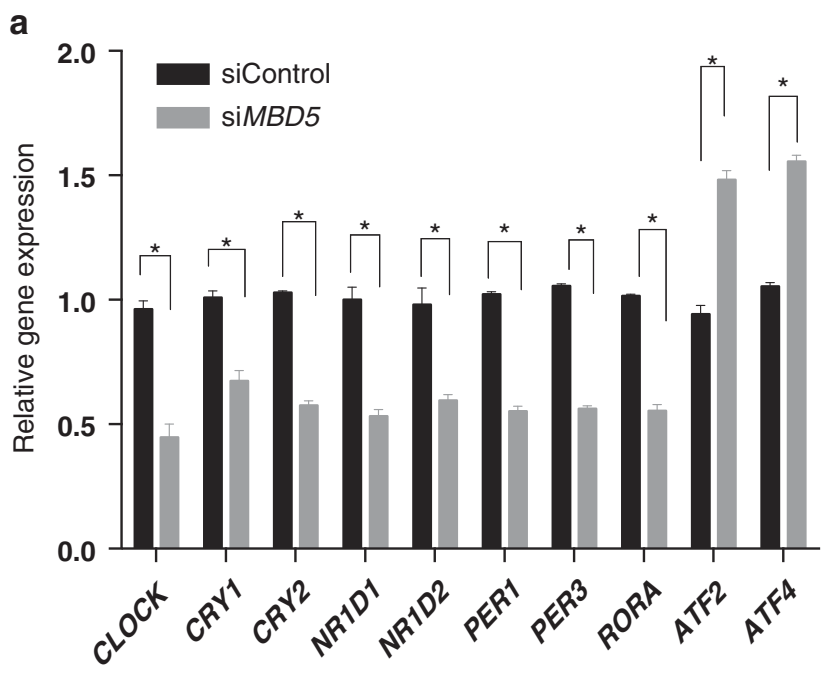

b

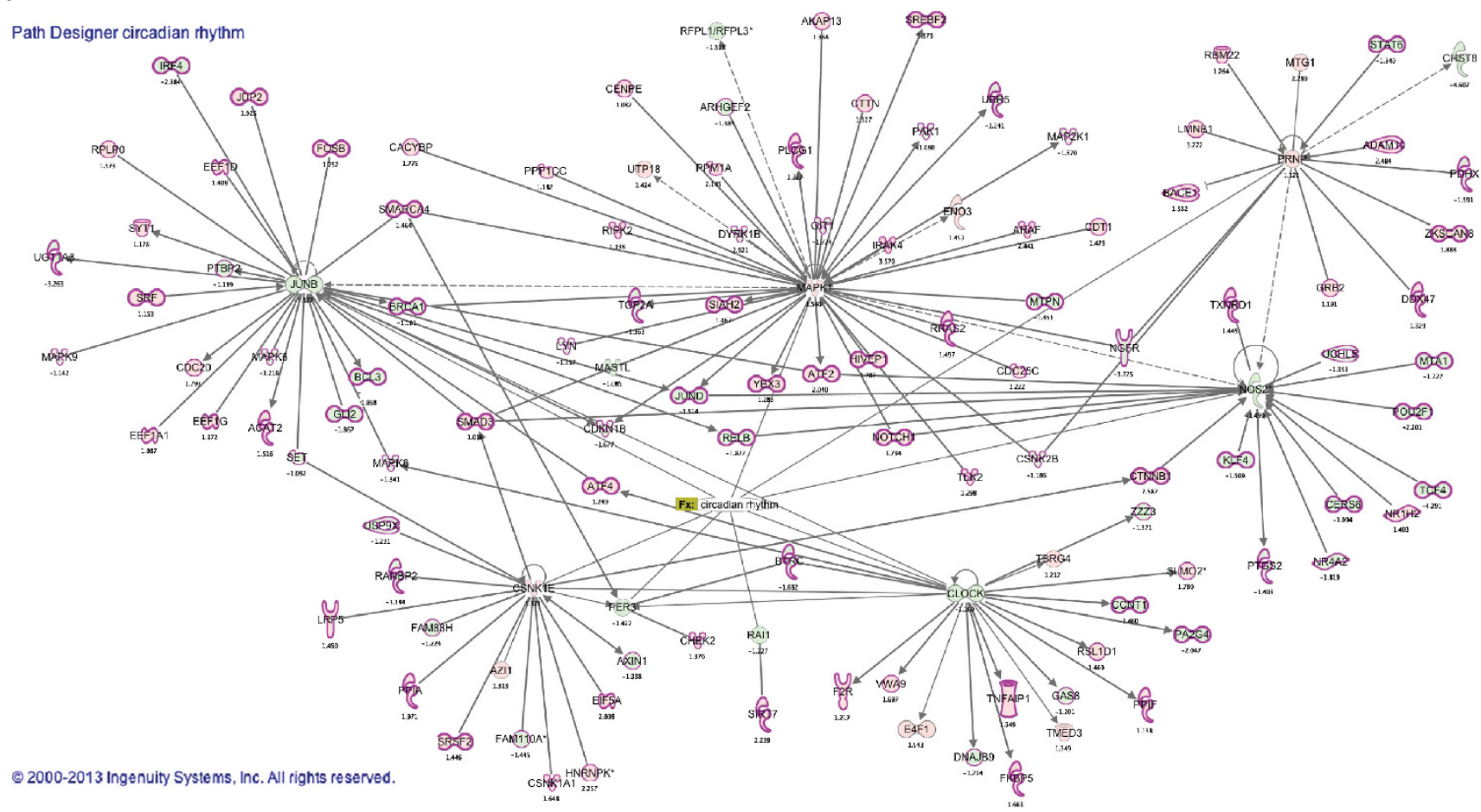

Figure 2 Knockdown of MBD5 is associated with dysregulation of key circadian rhythm genes and altered circadian signaling pathway. (a) Relative expression levels obtained from quantitative real-time PCR of circadian rhythm genes found dysregulated by microarray analysis of MBD5 siRNA knockdown cells are shown. Data are an average of three independent experiments performed in triplicate. Control siRNA is shown in black, and MBD5 siRNA is shown in gray. Bars represent SE. Quantification of the test gene was compared with that of the housekeeping gene GAPDH. Circadian genes that were altered in MBD5 knockdown microarray have altered expression in MBD5 siRNA knockdown SH-SY5Y cells. Asterisks indicate statistical significance $(P<0.001)$ as calculated with the Student's $t$-test and the Holm-Šídák test. (b) The pathway map of for circadian signaling $(P=0.00474)$ drawn using IPA was one of the top significantly enriched canonical pathways in MBD5 knockdown SH-SY5Y cell lines. Genes highlighted in red were upregulated, and genes in green were downregulated. The darker the color, the higher the fold change in gene expression. Values under each gene are the fold change observed in the study.

confound adequate assessment of the sleep problems in this syndrome, as medications may mask the reporting of specific sleep difficulties, limiting the extent of the data collected. More severe sleep issues may be present than were reported. Thus additional studies are required to more fully understand the specific problems in del 2q23.1 syndrome, including assessment of individuals across lifespan. Also, the molecular data presented here corroborate the sleep phenotypes present in del 2q23.1 syndrome and show that MBD5 has a role in circadian gene regulation.

\section{2q23.1 deletion syndrome, SMS, and FXS shared sleep pathway} Disease phenotypes are complex, resulting from the interaction of many biological pathways across development. Even the phenotypes that result from single-gene disorders are likely the result of the 

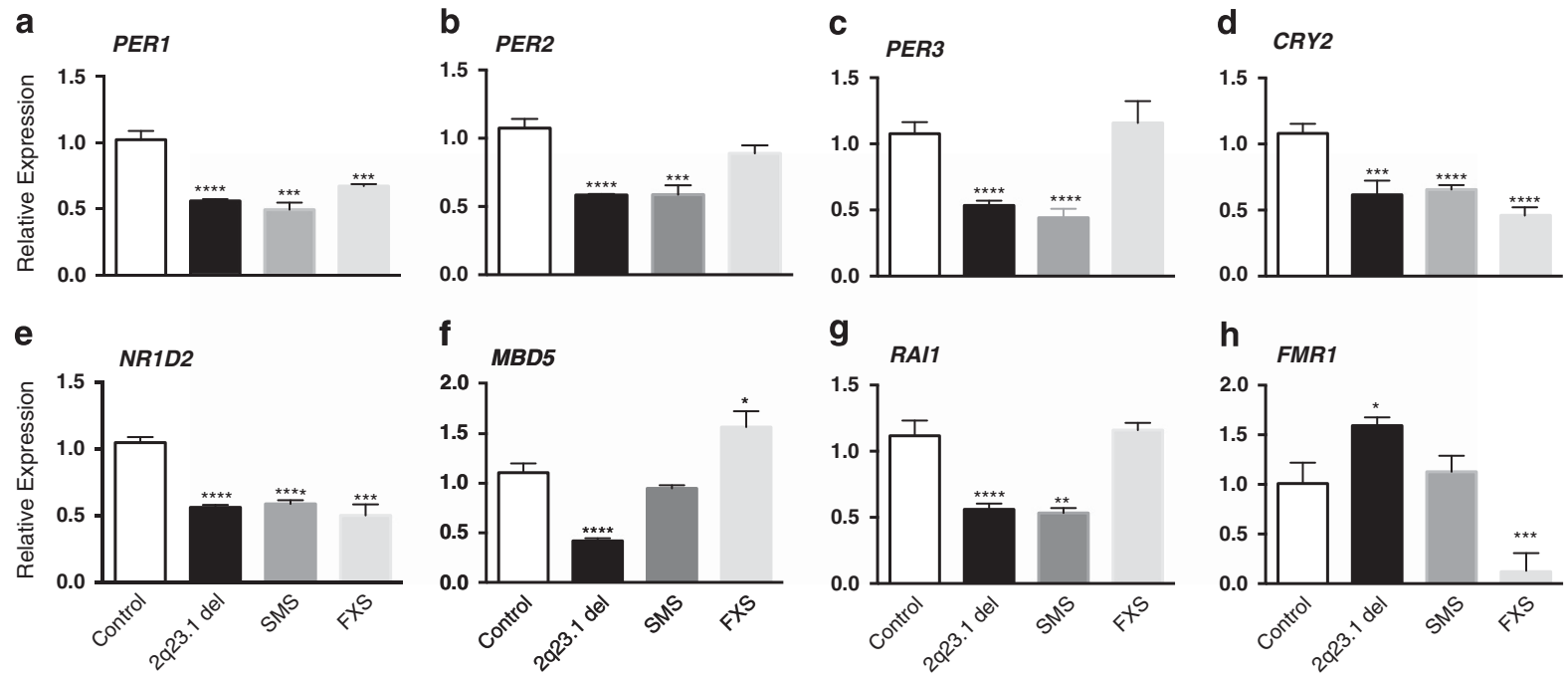

Figure 3 Gene dysregulation in del 2q23.1, Smith-Magenis, and fragile X syndrome cell lines suggests common molecular pathways. Quantitative real-time PCR of mRNA from LCLs from patients with SMS, FXS, or del 2q23.1 was performed. Data shown are calculated relative to mRNA expression levels from control subjects to assess expression of MBD5, RAI1, FMR1, PER1, PER2, PER3, NR1D2, and CRY1 across each syndrome. Data are an average of three independent experiments performed in triplicate. All expression values are calculated relative to control levels set at 1.0. Asterisks indicate significant differences between samples from patients versus control subjects $\left(* * * * P<0.0001,{ }^{*} * P<0.001\right.$, and $\left.* P<0.01\right)$. $P$-values were determined by unpaired $t$-test. Error bars represent the SD from the mean. (a) Although MBD5 expression is reduced in 2q23.1 deletion syndrome, overexpression is observed in FXS cell lines. (b) RAl1 expression is reduced in 2q23.1 deletion syndrome and SMS cell lines but not in FXS. (c) FMR1 expression is very low in FXS lines but overexpressed in 2q23.1 deletion syndrome cell lines, with normal expression in SMS lines. (d-f) Expression of PER1, PER2, and PER3 is impaired in 2q23.1 deletion and SMS lines but at normal levels in FXS cell lines. (g) NR1D2 expression is reduced in 2q23.1 deletion, SMS, and FXS lines. (h) CRY1 expression is reduced in 2q23.1 deletion, SMS, and FXS lines.

Table 4 Putative E box sites for MBD5, RAI1, and FMR1

\begin{tabular}{|c|c|c|c|}
\hline & Sequence $\left(5^{\prime}-3^{\prime}\right)$ & Genome coordinates (hg19) & $\begin{array}{c}\text { Location } \\
\text { upstream of } A T G\end{array}$ \\
\hline \multicolumn{4}{|c|}{ MBD5 } \\
\hline 1 & CACGTG & chr2.hg19:g.148778363_148778368 & -226 \\
\hline 2 & CAATTG & chr2.hg19:g.148777876_148777881 & -713 \\
\hline 3 & CAGCTG & chr2.hg19:g.148777454_148777459 & -1135 \\
\hline 4 & CAATTG & chr2.hg19:g.148776675_148776680 & -1914 \\
\hline 5 & CAATTG & chr2.hg19:g.148776650_148776655 & -1939 \\
\hline 6 & CAGCTG & chr2.hg19:g.148776217_148776222 & -2372 \\
\hline \multicolumn{4}{|c|}{ RAl1 } \\
\hline 1 & CAGCTG & chr17.hg19:g.17582772_17582777 & -2015 \\
\hline 2 & CAGCTG & chr17.hg19:g.17582365_17582370 & -2422 \\
\hline \multicolumn{4}{|c|}{ FMR1 } \\
\hline 1 & CAGCTG & chrX.hg19:g.147008609_147008614 & -579 \\
\hline 2 & CAGCTG & chrX.hg19:g.147008301_147008306 & -887 \\
\hline 3 & CAGCTG & chrX.hg19:g.147007263_147007268 & -1925 \\
\hline 4 & CAGCTG & chrX.hg19:g.147007055_147007060 & -2133 \\
\hline
\end{tabular}

a 2500 base pairs upstream from ATG of MBD5, RAI1, or FMR1.

interactions of multiple pathobiological processes that interact in a complex network. ${ }^{30}$ The disorders in this study, del 2q23.1, SMS and FXS, all clearly share common sleep phenotypes, such as difficulty falling asleep, shorter sleep duration, or night-waking episodes. ${ }^{31,32}$ Although RAI1 and FMR1 have been shown to regulate or affect expression of circadian rhythm genes, ${ }^{23,28}$ published sleep data suggest that SMS has a more severe sleep phenotype than FXS. ${ }^{32,33}$ In addition, from this study, SMS may have more similar sleep phenotype to del 2q23.1 than FXS because of the more frequent concerns for daytime sleepiness and napping. ${ }^{32,33}$

Our bioinformatics analyses identified potential E boxes that suggest that RAI1 and MBD5 could be circadianly regulated by components of the molecular clock through their E boxes. Further studies involving whether these $\mathrm{E}$ boxes actually function like typical E boxes are necessary to elucidate the role of the regulation of MBD5, RAI1, and FMR1 in circadian rhythm.

The expression data presented here demonstrate that RAI1, MBD5, and FMR1 likely function in overlapping pathways that may explain etiologies for sleep disturbance and other phenotypes in each disorder. Data suggest that RAI1 and FMR1 may be more molecularly connected to MBD5 than to one another. Supporting our above hypothesis is a recent study on specific molecular pathways and circuits in autism, where ASD and ID risk genes were mapped onto co-expression networks of developmental trajectories and transcriptional profiles in the fetal and adult cortical laminae. ${ }^{34}$ These studies showed bioinformatically that FMRP interacts with a series of ASD genes, one of them being MBD5. ${ }^{34}$

Finally, we show that the expression levels of NR1D2 and CRY2 were significantly reduced in 2q23.1 deletion syndrome, SMS, and FXS LCLs. Interestingly, loss of CRY2 has been linked to high anxiety, and NR1D2 was recently shown to protect normal metabolic function. ${ }^{35,36}$ Anxiety levels and abnormal metabolic function resulting in weight problems are two phenotypes present in these three disorders. The findings of dysregulation of circadian mRNA expression in connection with other phenotypes suggest that circadian rhythm defects not only contribute to sleep disturbances but also to other phenotypes as well. Overall, data suggest a potentially complex relationship for gene regulation across MBD5, RAI1, and FMR1, with key roles for each in the regulation of circadian gene expression. 
a

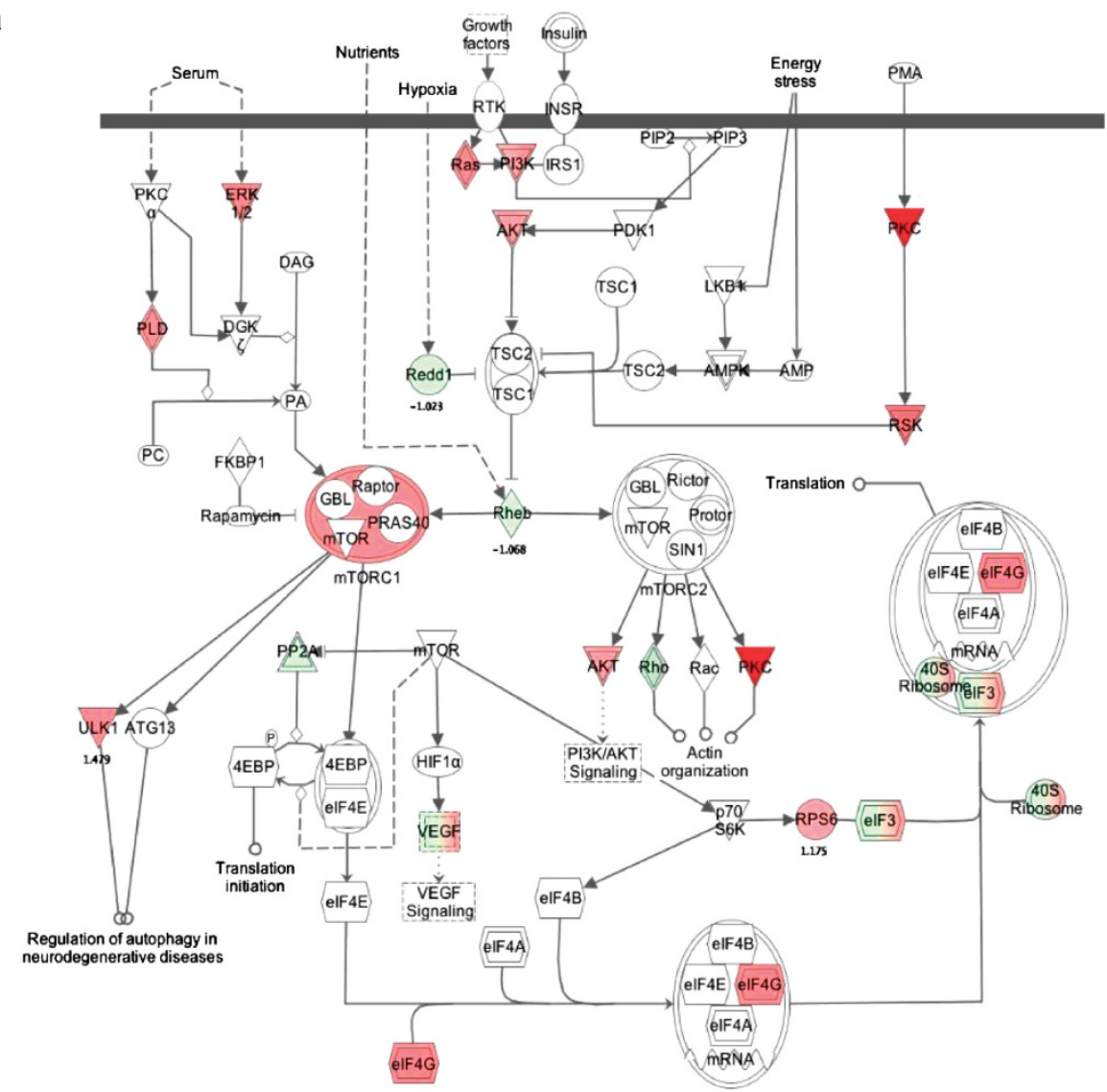

b

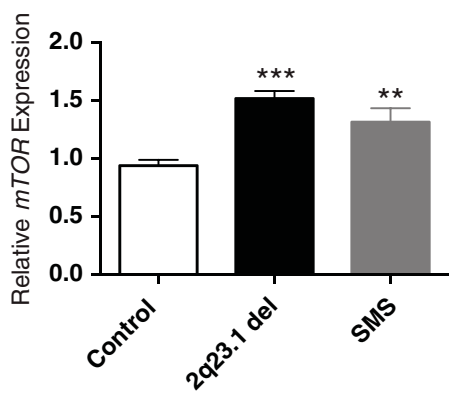

2000-2013 ingenuity Systems, inc. All nights reserved.

Figure 4 Dysregulation of mTOR signaling pathway and $m T O R$ in sleep phenotype of del 2q23.1 and SMS. (a) Significantly altered mTOR signaling pathway due to knockdown of MBD5 or RA/1. Illustration of a key pathway being altered by altered expression due to knockdown of MBD5 or RA/1 in SH-SY5Y cells (Ingenuity Pathway Analysis). The top altered pathway, mTOR signaling $(P=0.00329)$, has a role in proper sleep. Genes highlighted in green were downregulated in MBD5 or RAl1 in knockdown SH-SY5Y cells, and genes in red were upregulated in MBD5 or RAl1 in knockdown SH-SY5Y cells. The darker the color, the higher the fold change in gene expression. Values under each gene represent the fold change. (b) Significantly increased mRNA expression of mTOR in del 2q23.1 and SMS LCLs. Quantitative real-time PCR of mRNA from LCLs from patients with SMS and del 2q23.1 was performed. Data shown are calculated relative to mRNA expression levels from control subjects to assess the expression of $m T O R$ across each syndrome. Data are an average of three independent experiments performed in triplicate. All expression values are calculated relative to control levels set at 1.0. Asterisks indicate significant differences between samples from patients versus control subjects ( $* * * P<0.005$, and ${ }^{* *} P<0.05$ ). $P$-values were determined by unpaired $t$-test. Error bars represent the SD from the mean.

\section{MBD5, RAI1, FMR1, mTOR signaling pathway, and sleep disturbance}

Recently, the mTOR signaling pathway was associated with circadian rhythm. In humans, the master circadian clock is localized in the suprachiasmatic nucleus (SCN) of the hypothalamus. The circadian clock is thought to be reset by rapid induction of gene expression and, in particular, the expression of the circadian clock genes PER1 and PER2. ${ }^{37}$ In recent studies, mTOR-evoked mRNA translation alters the capacity of light to couple to the core clock timing mechanism, suggesting that the mTOR signaling pathway modulates photic entrainment of the SCN circadian clock. ${ }^{37}$ In addition, dysregulation of mTOR signaling is found in mouse and Drosophila models of FXS, which show that loss of FMRP leads to altered circadian rhythm behaviors and sleep-dependent synaptic renormalization. ${ }^{38}$ Also, in human studies, FXS patients show altered mTOR signaling. ${ }^{39}$ Moreover, these findings suggest that MBD5, RAI1, and FMR1 dysregulation could disrupt the $\mathrm{mTOR}$ signaling pathway, disrupting mammalian period (PER(1-2)) genes, which have key roles in photic entrainment of the circadian clock to light pulses. ${ }^{39}$ Recent studies in Fmr1/Fxr2 double KO mice show that absence of Fmrp/Fxr2p results in altered cyclical patterns of abundance of several core clock component mRNAs in the liver, including $\mathrm{Per}(1-2) .{ }^{28}$ Furthermore, FMRP and FXR2P together increase PER(1-2) mediated BMAL1Neuronal PAS2 (NPAS2) transcriptional activity, which is an essential component of circadian clock. ${ }^{28}$ Data shown here demonstrate dysregulation of some or all of the PER genes in 2q23.1 deletion syndrome, SMS, and FXS cases. Further study of the mTOR pathway in relation to sleep could be a key to identifying possible drug therapies to alleviate the sleep disturbances in these disorders. Finally, understanding the pathways and mechanisms in which genes disrupt neuronal development and brain function will allow us to understand the pathogenesis of phenotypes, such as sleep.

\section{CONFLICT OF INTEREST}

The authors declare no conflict of interest.

\section{ACKNOWLEDGEMENTS}

We are grateful to PRISMS and to the 2q23.1 Facebook group and all of the study participants and their families for their cooperation in this study. We thank the Fondation Jérôme Lejeune and the Smith-Magenis Syndrome 
Research Foundation for funding portions of this study. This work was supported, in part, by the resources from Virginia Commonwealth University, Baylor College of Medicine, and the Jan and Dan Duncan Neurological Research Institute at the Texas Children's Hospital.

1 Jiang $\mathrm{YH}$, Yuen RK, Jin X et al: Detection of clinically relevant genetic variants in autism spectrum disorder by whole-genome sequencing. Am J Hum Genet 2013; 93 : 249-263.

2 Kotagal S, Broomall E: Sleep in children with autism spectrum disorder. Pediatr Neurol 2012; 47: 242-251.

3 Malow BA, Adkins KW, Reynolds A et al: Parent-based sleep education for children with autism spectrum disorders. J Autism Dev Disord 2013; 44: 216-228.

4 Talkowski ME, Mullegama SV, Rosenfeld JA et al: Assessment of 2q23.1 microdeletion syndrome implicates MBD5 as a single causal locus of intellectual disability, epilepsy, and autism spectrum disorder. Am J Hum Genet 2011; 89: 551-563.

5 Mullegama SV, Rosenfeld JA, Orellana C et al: Reciprocal deletion and duplication at 2q23.1 indicates a role for MBD5 in autism spectrum disorder. Eur J Hum Genet 2014, 22: 57-63.

6 Noh GJ, Graham JM Jr: 2q23.1 microdeletion of the MBD5 gene in a female with seizures, developmental delay and distinct dysmorphic features. Eur J Med Genet 2012; 55: 59-62.

7 Chung BH, Stavropoulos J, Marshall CR, Weksberg R, Scherer SW, Yoon G: 2q23 De novo microdeletion involving the MBD5 gene in a patient with developmental delay, postnatal microcephaly and distinct facial features. Am J Med Genet A 2011; 155A: 424-429.

8 Williams SR, Mullegama SV, Rosenfeld JA et al: Haploinsufficiency of MBD5 associated with a syndrome involving microcephaly, intellectual disabilities, severe speech impairment, and seizures. Eur J Hum Genet 2010; 18: 436-441.

9 van Bon BW, Koolen DA, Brueton L et al: The 2q23.1 microdeletion syndrome: clinical and behavioural phenotype. Eur J Hum Genet 2010; 18: 163-170.

10 Jaillard S, Dubourg C, Gerard-Blanluet M et al: 2q23.1 microdeletion identified by array comparative genomic hybridisation: an emerging phenotype with Angelman-like features? J Med Genet 2009; 46: 847-855.

11 Bonnet C, Ali Khan A, Bresso E et al: Extended spectrum of MBD5 mutations in neurodevelopmental disorders. Eur J Hum Genet 2013; 21: 1457-1461.

12 Hodge JC, Mitchell E, Pillalamarri V et al: Disruption of MBD5 contributes to a spectrum of psychopathology and neurodevelopmental abnormalities. Mol Psychiatry 2014; 19: 368-379.

13 Cao R, Li A, Cho HY, Lee B, Obrietan K: Mammalian target of rapamycin signaling modulates photic entrainment of the suprachiasmatic circadian clock. J Neurosci 2010; 30: 6302-6314.

14 Buysse DJ, Reynolds CF 3rd, Monk TH, Berman SR, Kupfer DJ: The Pittsburgh Sleep Quality Index: a new instrument for psychiatric practice and research. Psychiatry Res 1989; 28: 193-213.

15 Johns MW: A new method for measuring daytime sleepiness: the Epworth sleepiness scale. Sleep 1991; 14: 540-545.

16 Truong HT, Solaymani-Kohal S, Baker KR et al: Diagnosing Smith-Magenis syndrome and duplication $17 \mathrm{p} 11.2$ syndrome by RAI1 gene copy number variation using quantitative real-time PCR. Genet Test 2008; 12: 67-73.

17 Slager RE, Newton TL, Vlangos CN, Finucane B, Elsea SH: Mutations in RAI1 associated with Smith-Magenis syndrome. Nat Genet 2003; 33: 466-468.

18 Vlangos CN, Yim DK, Elsea SH: Refinement of the Smith-Magenis syndrome critical region to approximately $950 \mathrm{~kb}$ and assessment of $17 \mathrm{p} 11.2$ deletions. Are all deletions created equally? Mol Genet Metab 2003; 79: 134-141.
19 Girirajan S, Vlangos CN, Szomju BB et al: Genotype-phenotype correlation in Smith-Magenis syndrome: evidence that multiple genes in $17 \mathrm{p} 11.2$ contribute to the clinical spectrum. Genet Med 2006; 8: 417-427.

20 Vincent A, Heitz D, Petit C, Kretz C, Oberle I, Mandel JL: Abnormal pattern detected in fragile-X patients by pulsed-field gel electrophoresis. Nature 1991; 349: 624-626.

21 Eichler EE, Holden JJ, Popovich BW et al: Length of uninterrupted CGG repeats determines instability in the FMR1 gene. Nat Genet 1994; 8: 88-94.

22 Gu Y, Lugenbeel KA, Vockley JG, Grody WW, Nelson DL: A de novo deletion in FMR1 in a patient with developmental delay. Hum Mol Genet 1994; 3: 1705-1706.

23 Williams SR, Zies D, Mullegama SV, Grotewiel MS, Elsea SH: Smith-Magenis syndrome results in disruption of CLOCK gene transcription and reveals an integral role for RAI1 in the maintenance of circadian rhythmicity. Am J Hum Genet 2012; 90: 941-949.

24 Girirajan S, Truong HT, Blanchard CL, Elsea SH: A functional network module for Smith-Magenis syndrome. Clin Genet 2009; 75: 364-374.

25 Burns B, Schmidt K, Williams SR, Kim S, Girirajan S, Elsea SH: Rai1 haploinsufficiency causes reduced Bdnf expression resulting in hyperphagia, obesity and altered fat distribution in mice and humans with no evidence of metabolic syndrome. Hum $\mathrm{Mol}$ Genet 2010; 19: 4026-4042.

26 Mullegama SV, Rosenfeld JA, Orellana C et al: Reciprocal deletion and duplication at 2q23.1 indicates a role for MBD5 in autism spectrum disorder. Eur J Hum Genet 2013; 22: 57-63.

27 Ueda HR, Hayashi S, Chen W et al: System-level identification of transcriptional circuits underlying mammalian circadian clocks. Nat Genet 2005; 37 : 187-192.

28 Zhang J, Fang Z, Jud C et al: Fragile X-related proteins regulate mammalian circadian behavioral rhythms. Am J Hum Genet 2008; 83: 43-52.

29 Glickman G: Circadian rhythms and sleep in children with autism. Neurosci Biobehav Rev 2010; 34: 755-768.

30 Barabasi AL, Gulbahce N, Loscalzo J: Network medicine: a network-based approach to human disease. Nat Rev Genet 2011; 12: 56-68.

31 Greenberg F, Lewis RA, Potocki L et al: Multi-disciplinary clinical study of Smith-Magenis syndrome (deletion 17p11.2). Am J Med Genet 1996; 62 : 247-254.

32 Kronk R, Bishop EE, Raspa M, Bickel JO, Mandel DA, Bailey DB Jr.: Prevalence, nature, and correlates of sleep problems among children with fragile $\mathrm{X}$ syndrome based on a large scale parent survey. Sleep 2010; 33: 679-687.

33 Boone PM, Reiter RJ, Glaze DG, Tan DX, Lupski JR, Potocki L: Abnormal circadian rhythm of melatonin in Smith-Magenis syndrome patients with RAI1 point mutations. Am J Med Genet A 2011; 155A: 2024-2027.

34 Parikshak NN, Luo R, Zhang A et al: Integrative functional genomic analyses implicate specific molecular pathways and circuits in autism. Cell 2013; 155: 1008-1021.

35 Bugge A, Feng D, Everett LJ et al: Rev-erbalpha and Rev-erbbeta coordinately protect the circadian clock and normal metabolic function. Genes Dev 2012; 26: 657-667.

36 De Bundel D, Gangarossa G, Biever A, Bonnefont X, Valjent E: Cognitive dysfunction, elevated anxiety, and reduced cocaine response in circadian clock-deficient cryptochrome knockout mice. Front Behav Neurosci 2013; 7: 152.

37 Cao R, Anderson FE, Jung YJ, Dziema H, Obrietan K: Circadian regulation of mammalian target of rapamycin signaling in the mouse suprachiasmatic nucleus. Neuroscience 2011; 181: 79-88.

38 Bushey D, Tononi G, Cirelli C: Sleep and synaptic homeostasis: structural evidence in Drosophila. Science 2011; 332: 1576-1581.

39 Hoeffer CA, Sanchez E, Hagerman RJ et al: Altered mTOR signaling and enhanced CYFIP2 expression levels in subjects with fragile X syndrome. Genes Brain Behav 2012; 11: 332-341.

Supplementary Information accompanies this paper on European Journal of Human Genetics website (http://www.nature.com/ejhg) 